\begin{tabular}{|c|c|c|}
\hline $\begin{array}{l}\text { PKS } \\
\text { PUBLIC } \\
\text { KNDLLEDGE } \\
\text { PROJECT }\end{array}$ & $\begin{array}{c}\text { REVISTA DE GEOGRAFIA } \\
\text { (RECIFE) } \\
\text { http://www.revista.uffe.br/revistageografia }\end{array}$ & $\begin{array}{l}\text { OJS } \\
\text { OPEN } \\
\text { JORNAL } \\
\text { SYSTEMS }\end{array}$ \\
\hline
\end{tabular}

\title{
CARACTERISTICAS DA PESQUISA EM GEOMORFOLOGIA FLUVIAL BRASILEIRA ENTRE OS ANOS 2000-2017: EVOLUÇÃO E ÁREAS DE INTERESSE
}

\author{
Mariana de Oliveira Henriques ${ }^{1}$; Rochele Tambosi da Silva ${ }^{2}$; Pedro Pádua Chagas ${ }^{3}$; \\ Filipe Tadashi Rodrigues Oura ${ }^{4}$; Gabriel Ferreira de Oliveira ${ }^{5}$; Raphael Nunes de Souza \\ Lima ${ }^{6}$; Monica dos Santos Marçal ${ }^{7}$.
}

\footnotetext{
${ }^{1}$ Universidade Federal do Rio de Janeiro. Email: marihenriques91@gmail.com

${ }^{2}$ Universidade Federal do Rio de Janeiro. Email: pretatambosi@gmail.com

${ }^{3}$ Universidade Federal do Rio de Janeiro.Email: pftchagas@gmail.com

${ }^{4}$ Universidade Federal do Rio de Janeiro.Email: filipetroura@gmail.com

${ }^{5}$ Universidade Federal do Rio de Janeiro.Email: gabrielferreira92@hotmail.com

${ }^{6}$ Universidade Federal do Rio de Janeiro. Email: raphaelgeo85@gmail.com

${ }^{7}$ Universidade Federal do Rio de Janeiro. Email: monicamarcal@ufrj.br
}

\begin{abstract}
RESUMO
No trabalho buscou-se conhecer melhor o desenvolvimento do campo de atuação da geomorfologia fluvial e, analisar as tendências de publicação no ramo nacional. A partir das revistas selecionadas, foi analisada a evolução da distribuição espacial das universidades onde se originaram os trabalhos; a região onde se localizam as áreas de estudos; e por fim, analisadas as escalas temporais (milenar, secular ou tempo presente) e espaciais das pesquisas. Foram classificadas as principais áreas de interesse dentro da geomorfologia fluvial que vêm sido pesquisadas no panorama nacional. Para isso, foi feita uma planilha no software Excel, tendo como base o recorte de dados referente à Revista Brasileira de Geomorfologia (RBG) e à Associação Brasileira de Recursos Hídricos (ABRH), sendo classificadas atualmente ambas como B2, no conceito Qualis da Capes. Os resultados vêm abordando análises sobre sistemas fluviais, processos de erosão e transporte e deposição de sedimentos.

Palavras Chaves: Geomorfologia Fluvial ; Revistas RBG e RBRH; Conceito Qualis da Capes.
\end{abstract}

\section{CHARACTERISTICS OF RESEARCH IN BRAZILIAN FLUVIAL GEOMORPHOLOGY BETWEEN THE YEARS 2000-2017: EVOLUTION AND AREAS OF INTEREST}

\begin{abstract}
In this paper, we sought to know better the development of the field of fluvial geomorphology and to analyze the trends on national publications. From the selected journals, we analyzed the evolution of the spatial distribution of the universities where the selected works originated; the regions where study areas are located; and finally, the time scales (millenarian, secular or present time) and spatial scales of the research were analyzed. Furthermore, we analyzed the main areas of interest within the river geomorphology that were researched in the national panorama. For this, a spreadsheet was made in the Excel software, based on data from the Brazilian Journal of Geomorphology (RBG) and the Brazilian Association of Water Resources (ABRH), being currently classified as $\mathrm{A} 2$ and B2, respectively, in the Qualis concept (Capes). The results are dealing with analysis on river systems, erosion processes and sediment transport and deposition.
\end{abstract}

Keywords: Fluvial Geomorphology; RBG e RBRH journals; Qualis da Capes concept. 


\section{INTRODUÇÃO}

O Grupo de Pesquisa em Geomorfologia Fluvial da UFRJ (Universidade Federal do Rio de Janeiro) desenvolve estudos desde o ano de 2000 e nos últimos quatro anos procura compreender a sua importância através de publicações entre áreas afins, em quais subáreas tem se destacado e qual a relação com as questões ambientais relacionadas à água e gestão dos recursos hídricos no cenário nacional. Tal motivação levou o grupo à apresentação de trabalhos sobre o tema em duas jornadas de iniciação científica no âmbito da UFRJ, na qual é possível observar a relevância desta área de pesquisa na atualidade.

Não se pretende considerar aqui que a abordagem sugerida seja definitiva e representativa desta área da geomorfologia brasileira ao longo dos anos, mas chamar a atenção para importantes investimentos à pesquisa científica em universidades e instituições pelo Brasil, com produções publicadas em periódicos reconhecidos cientificamente e ver de que forma se refletiram nas pesquisas em geomorfologia fluvial. Destaca-se, ainda, que as informações alcançadas possam ajudar a compreender o desenvolvimento do campo da geomorfologia fluvial e, sobretudo, situar as pesquisas do grupo no contexto das tendências nacionais.

Com base neste contexto, o presente trabalho pretende apresentar dados quantitativos sobre as características da pesquisa em geomorfologia fluvial brasileira nos últimos dezessete anos e, ao mesmo tempo, propor uma discussão em relação a sua importância no âmbito das questões ambientais relacionadas à gestão dos recursos hídricos. A partir de uma análise dos artigos publicados desde a década de 2000, foi possível caracterizar dentro da geomorfologia fluvial qual o panorama desta ao longo dos anos e, com isso, realizar uma observação da sua evolução até os dias atuais. Foi realizado um levantamento das publicações brasileiras, utilizando duas revistas de grande visibilidade no ramo, a Revista Brasileira de Geomorfologia (RBG) e a Revista Brasileira de Recursos Hídricos (ABRH), dos anos de 2000 a 2017.

Inicialmente para este trabalho, as publicações em geomorfologia fluvial foram comparadas com outras dentro do ramo da Geomorfologia Geral, onde foi analisado se havia uma tendência de crescimento ou decréscimo ao passar dos anos. Posteriormente, foi criada uma proposta de subdivisão da Geomorfologia Fluvial segundo áreas de interesse, encontradas nas publicações nesse recorte de tempo, sendo elas: (1) interferências antrópicas; (2) formas e processos; (3) sedimentos fluviais; (4) teórico/metodológico em Geomorfologia Fluvial; (5) sistema fluvial; (6) vegetação em bacias hidrográficas; (7) erosão em ambientes 
fluviais e (8) hidrologia. Também foi feita a análise comparativa do número de publicações da Revista Brasileira de Recursos Hídricos e da Revista Brasileira de Geomorfologia, de acordo com as áreas de interesse da Geomorfologia fluvial. Além disso, a pesquisa mensura as regiões do país que possuem a maior parte das publicações, além do gênero dos autores e, por fim, as escalas temporal e espacial das publicações.

\section{MATERIAL E MÉTODOS}

A metodologia aplicada para a pesquisa tem como base a revisão bibliográfica das publicações contidas na Revista Brasileira de Geomorfologia (RBG) e na Revista Brasileira de Recursos Hídricos. A seleção dos artigos escolhidos foi através da avaliação do Qualis da Capes, uma plataforma utilizada para avaliar as revistas e publicações científicas, no qual as notas vão de A1 a C. A escolha destas revistas justifica-se uma vez que estas são de grande visibilidade no ramo da Geomorfologia Fluvial. Inicialmente, o levantamento dos dados contidos é referente aos anos compreendidos entre 2000 e 2015, cujas revistas supracitadas tinham avaliações positivas do Qualis da Capes (A1).

Em seguida, foi realizada uma revisão dos dados levantados anteriormente e atualizados até o ano de 2017. Após a seleção das revistas, foi criada uma planilha no software Excel, contendo todas as publicações de 2000 a 2017. Essa planilha foi tabelada em variáveis que continham o nome do artigo, a data da publicação, a revista no qual foi publicada, o gênero e a instituição do primeiro, segundo e terceiro autor, a escala espacial, a escala temporal e a área de estudo. A partir destes dados, foram criadas tabelas comparativas, mostrando proporcionalmente os resultados referentes a gênero dos autores (homem/mulher), a escala espacial das publicações (bacia, canal, entre outras), a escala temporal (dezenas, centenas, milhares ou milhões de anos) e as regiões brasileiras de onde se originaram as publicações. Seguidamente, foi feita a proposta da criação de áreas de interesse na Geomorfologia Fluvial a partir dos temas mais vistos dentro das publicações analisadas.

As áreas com uma maior quantidade de publicação foram: Influências Antrópicas (impactos antrópicos, regulação de rio, mudança de canal, etc.), Sedimentação Fluvial (caracterização e transporte), Teórico e Metodológico em Geomorfologia Fluvial, Sistema Fluvial, Formas e Processos, Vegetação em Bacias Hidrográficas, Erosão em ambientes fluviais (com destaque para esse tipo de processo) e Hidrologia. A partir dessa subdivisão foram geradas as áreas de interesse de maior incidência de publicações e ocorreu a tentativa de entender o porquê do destaque de cada uma delas. A partir desta distribuição, os artigos 
encontrados na área da Geomorfologia Fluvial dos anos 2000 a 2017 também foram divididos e transformados em tabelas comparativas de acordo com as áreas de interesse citadas anteriormente.

\section{RESULTADOS E DISCUSSÕES}

A Revista Brasileira de Recursos Hídricos (RBRH) tem sua sede na cidade de Porto Alegre, no Rio Grande do Sul, com seu primeiro volume datado de 1996. É uma revista publicada em sua maior parte por engenheiros especializados nas áreas florestais, ambientais, saneamento e principalmente hidrologia aplicada, na qual se destacam pesquisas relacionadas à dinâmica fluvial atual e direcionado às intervenções na paisagem. Já a Revista Brasileira de Geomorfologia (RBG), que também tem sua sede na cidade de Porto Alegre, no Rio Grande do Sul com o seu primeiro volume também no ano de 1996, tem um histórico de publicações por parte de geógrafos com trabalhos na área da geografia física, sobretudo na área de geomorfologia histórica e funcional da paisagem, bacias hidrográficas e canais fluviais (Figura 1).

Figura 1: Número de publicações por área de interesse em Geomorfologia Fluvial (Por revista; 2000-2017; Números absolutos).

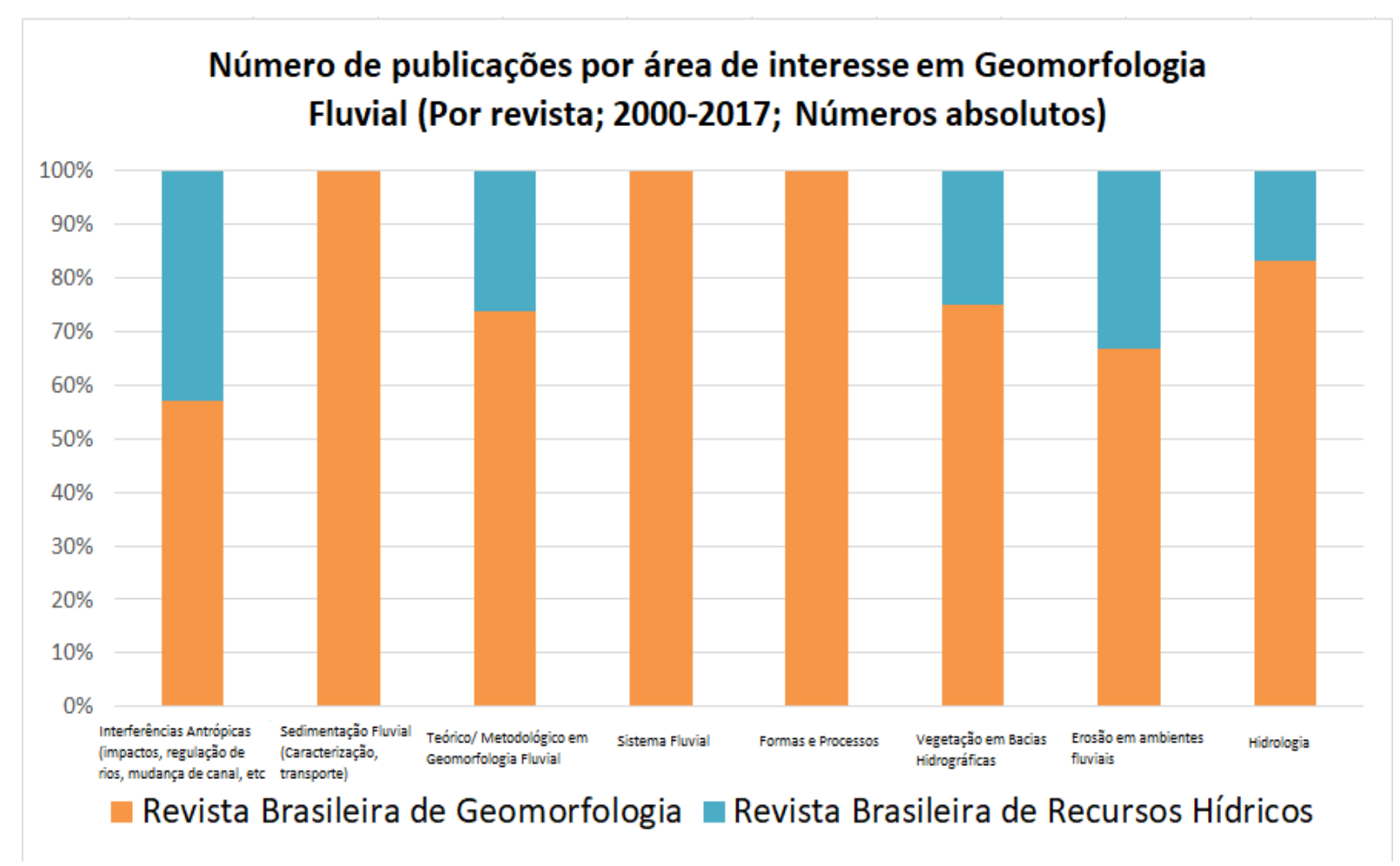


Entre os anos de 2000 a 2017, a revista RBRH publicou um total de 1.070 artigos, na qual 556 pertencem ao campo da Engenharia, sendo que onze trabalhos são voltados para a área da geomorfologia fluvial e caracterização e análises de bacias hidrográficas. No caso da revista RBG, de um total de 419 artigos publicados, 80 estão relacionados ao tema da geomorfologia fluvial, bacias hidrográficas e dinâmicas fluviais.

As áreas de interesse na geomorfologia fluvial foram criadas a partir da maior incidência de temas nos artigos encontrados nas duas revistas selecionadas. A primeira área destacada é referente ao tema de Interferências Antrópicas, no qual os artigos gerados discutem sobre a regulação de canais, impactos gerados pelo homem e qualquer outra alteração no sistema fluvial relacionada ao ser humano. É possível notar na Figura 1 que esta área de interesse foi a com maior número de publicações por parte da Revista Brasileira de Recursos Hídricos, lembrando que a RBRH é uma revista escrita em sua maior parte por engenheiros.

A segunda área destacada, que é muito presente no ramo da Geografia, é sobre Formas e Processos, sendo o tema com o maior número de publicações, 25 no total de 89 em ambas as revistas, como pode ser visto na Figura 2 na qual todas foram feitas pela revista RBG. Devido a sua importância nos estudos geográficos é compreensível que a RBG, sendo esta majoritariamente escrita por geógrafos, tenha tido $100 \%$ das publicações nessa área de interesse. A terceira área destacada é referente à Sedimentação Fluvial, sendo um tema muito recorrente entre os artigos, principalmente no contexto da caracterização e transporte de sedimentos. Tendo em vista que esta área poderia estar caracterizada como Formas e Processos, é notável seu grande destaque através da quantidade de artigos publicados referentes a este tema, dez artigos no total, sendo um número representativo se compararmos proporcionalmente com o total de artigos na área de interesse "Formas e Processos". 
Figura 2: Número de publicações por área de interesse em Geomorfologia Fluvial (Ambas as revistas; 2000-2017; Números Absolutos).

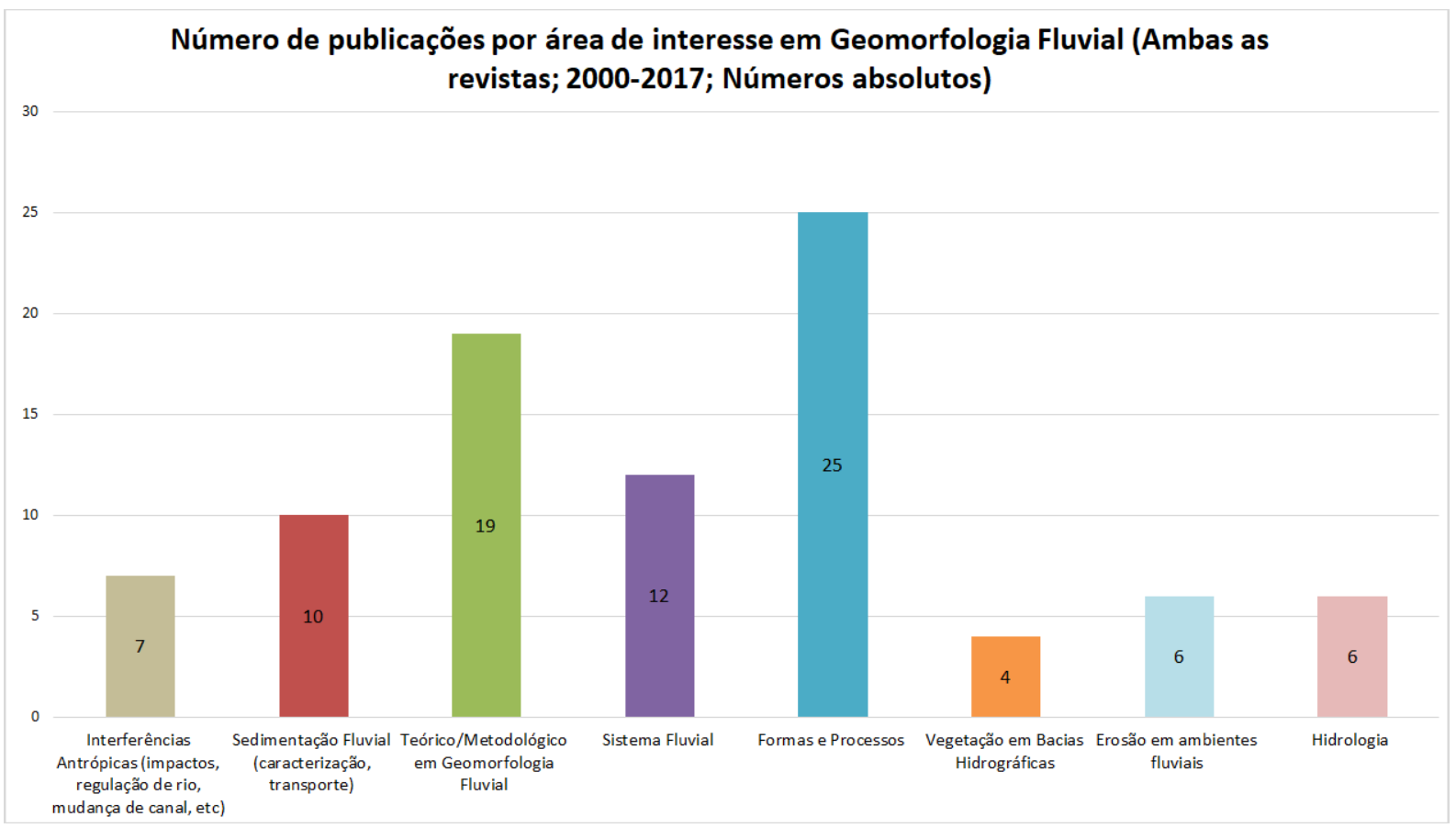

O mesmo ocorre com o quarto tema selecionado Erosão em Ambientes Fluviais, sendo este também uma área relacionada ao estudo de Processos e Formas, que merece destaque, pois tem publicações por ambas às revistas, principalmente pela revista RBG, essa área de interesse teve um total de seis publicações, justamente por erosão ser, sem dúvidas, um dos processos mais estudados e, consequentemente, ocorrentes na dinâmica geomorfológica. A quinta área destacada esta relacionada ao estudo Teórico/Metodológico em Geomorfologia Fluvial, sendo esta a área de interesse com o segundo maior número de publicações, dezenove no total, é um assunto muito importante, pois trata de novas ferramentas e métodos para facilitar a ampliação dos estudos em Geomorfologia Fluvial, nos quais foram encontrados artigos que sugerem ferramentas como softwares novos.

A sexta área de destaque refere-se ao Sistema Fluvial o qual é bastante recorrente em artigos que apresentam explicações sobre o sistema, através de estudos de caracterização. É importante destacar que este tema tem todas as suas publicações realizadas pela revista RBG. Com um total de doze artigos, de acordo com a Figura 2, é um tema indispensável na dinâmica da Geomorfologia Fluvial. A sétima área selecionada está relacionada à Vegetação em Bacias Hidrográficas, e tem como característica explicar a influência da vegetação na dinâmica dos processos fluviais. Foram encontrados quatro artigos que tratavam exclusivamente desse assunto. Por fim, a oitava área de interesse é relacionada aos estudos 
sobre Hidrologia, sendo este um tema recorrente, principalmente para caracterização e entendimento de regimes hidrológicos. É possível notar na Figura 2 um total de seis artigos com esse tema.

Dentro de todos os artigos encontrados na área de Geomorfologia Fluvial, especificamente nas revistas da RBRH e na RBG, buscou-se analisar nesta pesquisa as regiões de origem de cada artigo publicado. Além disso, buscou-se avaliar como o estudo da escala espacial das publicações (bacia, drenagem, canal), a escala temporal das publicações (eventos de anos, décadas, centenas, milhares e milhões de anos), o gênero dos autores nas publicações e como estes estão distribuídos. Na análise de comparação (de acordo com a Figura 3) entre as duas revistas selecionadas para esta pesquisa é possível notar a falta de publicações por parte da revista RBRH nas regiões Centro-Oeste e Norte, além de publicações no exterior. E por mais que esta revista tenha um baixo número de publicações no Brasil, destaca-se suas publicações predominantemente nas regiões sul e nordeste.

Figura 3: Distribuição das publicações das revistas RBRH e RBG por região onde foram publicadas (entre os anos de 2000-2017).

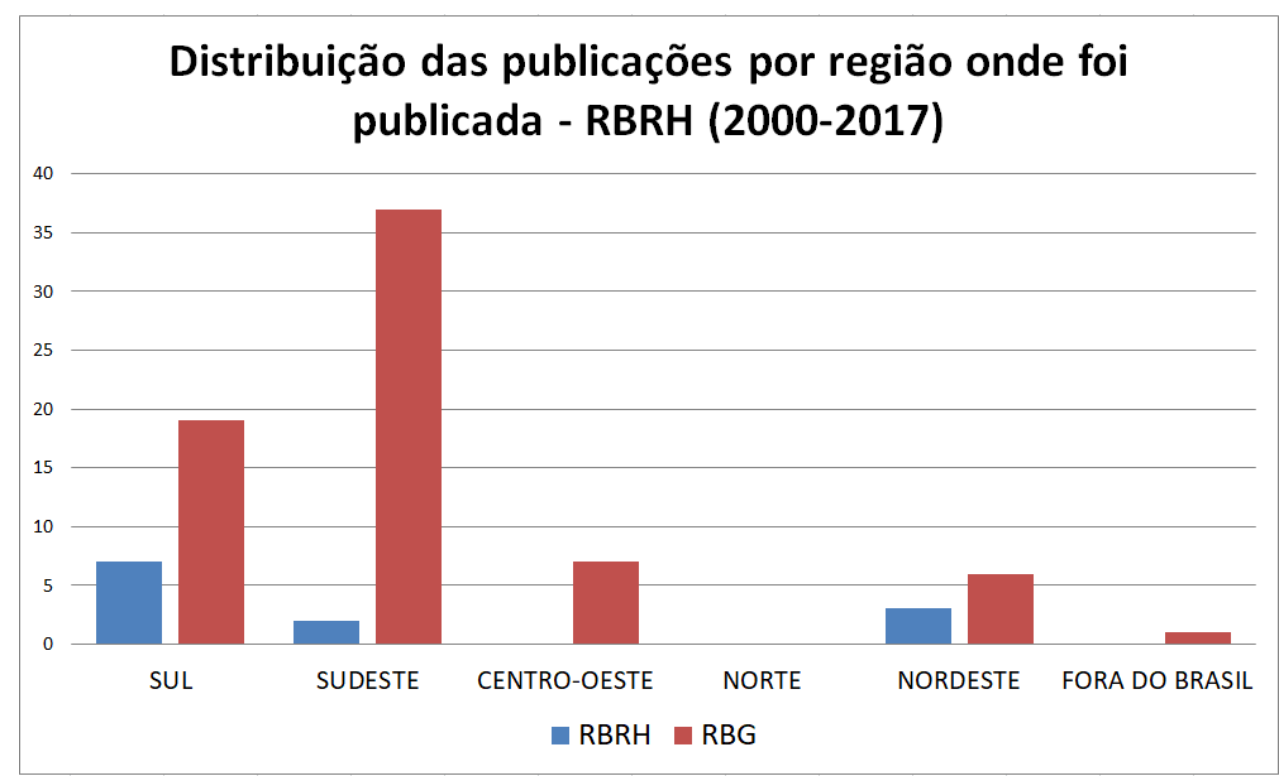

Na região sul do país a revista RBRH tem um total de sete publicações, na região nordeste conta com um total de três publicações e na região sudeste com duas publicações. Já na revista RBG, é notável um maior número de publicações no qual grande parte é oriunda da região sudeste do Brasil com um total de trinta e sete publicações. A segunda região com 
maior número de publicações pela RBG é a região sul com um total de dezenove artigos publicados. Na região centro-oeste foi contabilizado um total de sete publicações e na região nordeste um total de seis publicações contidas na revista. Por fim, também foi constatada uma publicação internacional da revista RBG. A partir da análise das pesquisas realizadas também foi possível constatar a quantidade de publicações que trabalhavam com a escala espacial de bacias ou canais dentre os anos 2000 e 2017.

Foram identificados oito artigos referentes às análises de bacia hidrográfica e quatro artigos em escalas de canais na revista RBRH. Ainda sobre os dados levantados desta revista, também foi observado que as escalas temporais dos eventos estudados contabilizam o total de oito publicações relacionados à eventos anuais, quatro publicações referentes à eventos de décadas e, por fim, cinco publicações alusivas a eventos que tem centenas de anos. Em relação a quantidade de publicações por gênero foi averiguado que foi contabilizado dezenove autores do gênero masculino e cinco autoras do gênero feminino do total analisado para o período da pesquisa. Após a verificação das publicações referentes à Revista RBG buscou-se nas pesquisas da geomorfologia fluvial analisar a escala espacial dos fenômenos pesquisados. As publicações contidas na revista RBG compreendem apenas uma publicação sobre rede de drenagem, cinquenta e três publicações sobre canais, uma publicação sobre sistemas fluviais e, por fim, vinte e duas publicações que trabalham à partir da escala de bacias hidrográficas.

No que se concerne a escala temporal dos eventos estudados nesta revista (RBG) foi constatado o total de vinte e duas publicações referentes a fenômenos anuais, seis publicações relacionados a fenômenos que ocorrem em um período de uma década aproximadamente, vinte publicações sobre fenômenos que ocorrem em centenas de anos, vinte e seis publicações referentes a eventos de milhares de anos e três eventos referentes a eventos de milhões de anos. Em relação a quantidade de publicações por gênero, sessenta e oito das publicações são de autores do gênero masculino e vinte e três autoras do gênero feminino.

\section{CONSIDERAÇÕES FINAIS}

Observou-se que com o passar dos anos, desde o início das pesquisas no ano 2000, há uma tendência de crescimento de publicações no ramo da Geomorfologia Fluvial e que principalmente a partir do ano de 2010 houve um aumento considerável das publicações, o que se pode relacionar, possivelmente, com a crise hídrica na região Sudeste no ano de 2012, no qual explica o maior número de artigos com origem na região Sudeste, como visto na 
Figura 3. Também é possível destacar a importância do uso de um banco de dados a partir deste material como referência para futuras pesquisas na área, como uma forma de filtro que facilita o encontro de determinados assuntos e palavras-chaves de acordo com as áreas de interesse propostas.

É de grande importância destacar, também, a contribuição da Geomorfologia Fluvial tanto em revistas ligadas à Geomorfologia quanto em revistas ligadas à outros temas. Podemos considerar como exemplo a Revista Brasileira de Recursos Hídricos (RBRH), que é caracterizada por ser uma revista da área de engenharia e se encontra integrada com a Geomorfologia Fluvial em diversos artigos, principalmente em temas relacionados à interação com a sociedade.

\section{REFERÊNCIAS}

REF VITTE, Antonio. A construção da geomorfologia no Brasil. Revista Brasileira da Geomorfologia. V.12, $\mathrm{n}^{\mathrm{O}} \quad 3 \quad$ (2011). Disponível em: http/www.lsi.unb.br/rbg/index.php?journal=rbg\&page=article\&op=view\&path $\% 5 B \% 5 \mathrm{D}=262$ \&path\%5B\%5D=198

COLTRINARI, Lylian. Geomorfologia: Caminhos e Perspectivas. Revista Brasileira da Geomorfologia. V.1, $\mathrm{n}^{\circ} 1 \quad$ (2000). Disponível http://www.lsie.unb.br/rbg/index.php?journal=rbg\&page=article\&op=view\&path\%5B $\% 5 \mathrm{D}=6$ $8 \&$ path $\% 5 \mathrm{~B} \% 5 \mathrm{D}=60$

WOHL, Ellen. Time and the rivers flowing: Fluvial geomorphology since 1960. Geomorphology. $\quad$ V. $216 . \quad$ Disponível em: https://www.researchgate.net/publication/262110976_Time_and_the_rivers_flowing_Fluvial_ geomorphology_since_1960

OLIVEIRA, Carmélia; SALGADO, André. Geomorfologia Brasileira: Panorama Geral da Produção Nacional de Alto Impacto no Quinquênio Entre 2006 e 2010. Revista Brasileira de $\begin{array}{llllllll}\text { Geomorfologia. } & \text { V. } & 14, & \mathrm{n}^{\circ} & 1 & \text { (2013). } & \text { Disponível em }\end{array}$ :http://www.lsie.unb.br/rbg/index.php?journal=rbg\&page=article\&op=view\&path\%5B\%5D= 421\&path\%5B\%5D=311

THORNDYCRAFT, Varyl. Fluvial Geomorphology: A perspective on current status and methods. Geomorphology, Vol. 98, 06.2008. Disponível em: https:/pure.royalholloway.ac.uk/portal/em/publications/fluvial-geomorphology-a-perspectiveon-current-status-and-methods(08c5439d-96ad-4e33-ada6-c9e7a8e98873).html 
PIÉGAY, Hervé. Trends in publications in fluvial geomorphology over two decades: A truly new era in the discipline owing to recente technological revolution. Geomorphology. 2015. Disponível em: http://acemap.sjtu.edu.cn/paper/paperpage?PaperID=3BBAF5CD STOTT, Tim, Review of research in fluvial geomorphology 2010-2011. 